\title{
IOS 2.0: new aspects on inter-organizational integration through enterprise 2.0 technologies
}

\author{
Christy M. K. Cheung ${ }^{1}$ • Martin Mocker ${ }^{2,3}$ • Daniel Schlagwein ${ }^{4}$ Ali Sunyaev ${ }^{5}$. \\ Klaus Turowski ${ }^{6}$
}

Published online: 9 October 2015

(C) Institute of Information Management, University of St. Gallen 2015

This special theme of Electronic Markets focuses on research concerned with the use of social technologies and "2.0" principles in the interaction between organization (i.e., with "inter-organizational systems (IOS) 2.0"). This theme falls within the larger space of Enterprise 2.0 research, but focuses in particular on inter-organizational use (between enterprises), not intra-organizational use (in a single enterprise). While there is great interest in practice regarding the use of 2.0 technologies to support intra-organizational communication, collaboration and interaction, information systems (IS) research has largely been oblivious to this important use of social technologies.

Before introducing the papers that make up this special issue, we briefly review the history of the use of " 2.0 " terms and concepts, and conceptualize "IOS 2.0 " as the emerging type of systems that are both "social" and "intra-organizational".

Ali Sunyaev

sunyaev@wiso.uni-koeln.de

$1 \quad$ Hong Kong Baptist University,

Hong Kong, Hong Kong

2 Reutlingen University, Reutlingen, Germany

3 MIT,

Cambridge, MA, USA

4 UNSW Australia Business School, Sydney, Australia

5 University of Cologne, Cologne, Germany

6 University of Magdeburg, Magdeburg, Germany

Web 2.0

In the early 2000s, new "social" applications and technologies emerged on the Internet (Shirky 2003). These social technologies included early social networking sites (e.g. Friendster launched in 2002), blogs (e.g., Blogger launched in 1999), photo sharing (Flickr launched in 2004), wikis (e.g., Wikipedia launched in 2001) and peer-to-peer file-sharing tools (e.g., Napster launched in 1999).

The term "web 2.0" was popularized by the "Web 2.0 Conference" in 2004. The "2.0" of the web 2.0 indicates a "second generation" Internet (similar to how versioning a software 2.0 indicates a major new generation). The notion of web 2.0 was based on an evidential shift in the way people used and conceptualized the Internet at that time. According to a "web 1.0" view, the Internet was something that end-users "read". According to a "web 2.0 " view, the Internet was (and is) something that end-users "read and write". As such, web 2.0 refers to a holistic generation change in the way that people interpret the Internet and in the prevailing culture around the Internet (values, culture, business models). (See further O'Reilly 2005; O'Reilly and Battelle 2009).

Later, with the extreme popularity of Facebook and Twitter towards the end-2000s, the term "social media" became en vouge. Social media is a narrow concept compared to web 2.0 , referring particularly to social networking sites and services (Facebook, Twitter, Instagram, Tumbler, WeChat, etc.) but it does not include other types of 2.0 technologies (e.g., mashups, folksonomies or peer-to-peer file-sharing services) and it does not include a notion of "shift" in values and culture that was part of the definitions of web 2.0. Nonetheless, social media has been proven the more popular concept and term, arguably because it is less "techy" and focuses on the end-user view of having alternative media to socially communicate at 
their hands. (See further Boyd and Ellison 2007; Kaplan and Haenlein 2010).

\section{Enterprise 2.0}

Enterprises and other organizations use social media to communicate to external audiences (typically their customers or the public in general). In the early days of web 2.0, many organizations were more concerned about the loss of business secrets on external social media through inappropriate communication by employees on platforms such as Facebook leading to various forms of "employee social media guidelines" (rather than encouraging their use). However, the vast majority of large organizations have since adopted social media in the time of $2005-10$ as a form of communicating externally (Schlagwein and Prasarnphanich 2014).

Furthermore, organizations have internally adopted these technologies for business-oriented communication and collaboration, a phenomenon dubbed "enterprise 2.0" (McAfee 2006). Enterprise 2.0 makes use of web 2.0 technologies such as blogs, microblogs and wikis inside the organization. For example, blogs allow the CEO to communicate to the whole enterprise, reducing separateness of top management and increasing transparency (Thompson 2007). Micro-blogging services such as Yammer offer similar functionality and usability to Twitter (Riemer et al. 2010). Wikis can be used as lightweight alternatives to traditional knowledge management systems (Levy 2009). Enterprise 2.0 has been of great interest to IS researchers (see further Leonardi et al. 2013; Richter et al. 2011; Williams et al. 2013).

\section{Towards "inter-organizational systems 2.0"}

Enterprise 2.0 has always included in its very conceptualization the use of social technologies between organizations (McAfee 2009). While such inter-organizational use took longer than other forms of enterprise 2.0 to emerge (possibly due to even higher cultural challenges, see Steinhueser et al. 2015, in this special theme), the inter-organizational part of enterprise 2.0 approaches provides many opportunities for more efficient collaboration (e.g., McKinsey Global Institute
2012). Despite the increasing relevance in practice, we are lacking a good empirical and theoretical understanding of inter-organizational enterprise 2.0.

Information systems that span between and across organizations, IT-enabled systems for inter-organizational integration, have traditionally been called "inter-organizational systems (IOS)" . The IOS term is used to distinguish such systems from intra-organizational systems. Examples of IOS include systems for supply chain management (SCM) or electronic data interchange (EDI). Both enterprise systems and IOS have been of great interest to the IS research community since the $1990 \mathrm{~s}$ (Hirschheim and Klein 2012).

IOS 2.0 can be seen as either the "next generation" of IOS and/or the use of web 2.0 and enterprise 2.0 principles in an inter-organizational context. Hence, to introduce the reader to the scope of the special theme, we distinguish between different types of information systems in Table 1 according to two dimensions.

In Table 1, the rows labelled "non-social/1.0" and "social/2.0" are to distinguish if the type of information system is commonly considered to be of a social and 2.0 nature. The columns labelled "intra-organizational" and "inter-organizational" are to distinguish between the uses of the information systems within or between organizations, respectively. Enterprise Systems (ES) such as ERP systems used within one organization fall into the type of non-social intra-organizational systems (some ERP systems can be used inter-organizationally as well). Inter-Organizational Systems (IOS) such as traditional SCM systems fall into the type of non-social intra-organizational systems. Internal Enterprise 2.0 systems (ES 2.0) such as enterprise blogs, microblogging or wikis are social, intra-organizational systems. Following this rationale, systems that replace or complement IOS for inter-organizational use and have substantial social functionality and other 2.0 characteristics are then "IOS 2.0".

While the above table delineates our conceptualization of IOS 2.0, the term is not clearly defined in practice or research as of yet. Hence, we have been inclusive (i.e., not rejecting papers as out of scope for this special issue) in allowing authors to express alternative definitions and understandings of how IOS 2.0 are to be conceptualized and what the term means to them.
Table 1 Types of information systems used in an enterprise/ organizational context

\begin{tabular}{llc}
\hline & Intra-organizational & Inter-organizational \\
\hline Non-social/1.0 & Enterprise Systems (ES) such as & Inter-Organizational Systems (IOS) \\
& ERP systems. & such as traditional SCM systems. \\
Social/2.0 & Internal Enterprise 2.0 Systems (ES 2.0) & "Inter-Organizational Systems \\
& such as enterprise microblogging systems. & 2.0 (IOS 2.0)" \\
\hline
\end{tabular}




\section{Papers in this special issue}

This special theme on "IOS 2.0" includes three papers.

Steinhueser, Richter and Smolnik's paper (Steinhueser et al. 2015), How to Bridge the Boundary? Determinants of Inter-Organizational Social Software Usage, transfers a theoretical model from the context of IOS 1.0 to the context of IOS 2.0 , putting its applicability to the test in a literature review and interview study. The model emphasizes the increased need for negotiation between the different participants in an IOS setting. According to the analysis, culture plays a significant role for IOS 2.0 while it was not considered a significant factor for the IOS 1.0 version of the model.

Setia, Richardson and Smith's paper (Setia et al. 2015), Business Value of Partner's IT Intensity: Value Co-Creation and Appropriation Between Customers and Suppliers, focuses on customer-supplier dyads. Based on the quantitative analysis of data from over 5000 dyads their research finds that increased use of Enterprise 2.0 technologies, as part of the general IT portfolio, by either partner in the dyad can help both partners create and appropriate value for each other.

Schneider and Sunyaev's paper (Schneider and Sunyaev 2015), CloudLive: A Life Cycle Framework for Cloud Services, develops a life-cycle framework for the sourcing of cloud-based services that enable the integration of IOS utilizing Enterprise 2.0 technology. Based on a literature analysis and expert interviews, their article identifies challenges for each stage of the life cycle as well as ways to address these challenges from both the cloud service customer and the cloud service provider perspective. This paper was reviewed through a regular Electronic Market review process, not by the special issue editors and reviewers, because one of the co-authors is also a co-editor of this special issue.

The three papers add knowledge to the emerging domain of Enterprise 2.0 tool use in an inter-organizational context and hopefully provide encouragement and a basis for future research.

\section{Dedication}

We dedicate this special issue to Holger Schrödl and his family. Holger initiated and led this special issue. His tragic and unexpected passing deeply saddened all of us and reminded us of how precious and fragile life is. We miss Holger and we will remember him as an amiable colleague and friend.

\section{References}

Boyd, D. M., \& Ellison, N. B. (2007). Social network sites: definition, history and scholarship. Journal of Computer-Mediated Communication, 13(1), 210-230.

Hirschheim, R., \& Klein, H. K. (2012). A glorious and not-so-short history of the information systems field. Journal of the Association for Information Systems, 13(4), 1-48.

Kaplan, A. M., \& Haenlein, M. (2010). Users of the world, unite! The challenges and opportunities of social media. Business Horizons, 53(1), 59-68.

Leonardi, P. M., Huysman, M., \& Steinfield, C. (2013). Enterprise social media: definition, history and prospects for the study of social technologies in organizations. Journal of Computer-Mediated Communication, 19(1), 1-19.

Levy, M. (2009). Web 2.0 implications on knowledge management. Journal of Knowledge Management, 13(1), 120-134.

McAfee, A. P. (2006). Enterprise 2.0: the dawn of emergent collaboration. MIT Sloan Management Review, 47(3), 21-28.

McAfee, A. P. (2009). Enterprise 2.0: new collaborative tools for your organization's toughest challenges. Harvard: Harvard Business Press.

McKinsey Global Institute (2012). The social economy: Unlocking value and productivity through social technologies. Report.

O'Reilly, T. (2005). What Is Web 2.0: Design patterns and business models for the next generation of software. http://oreilly.com/ web2/archive/what-is-web-20.html, Accessed 31 Aug 2015.

O'Reilly, T., \& Battelle, J. (2009). Web squared: Web 2.0 five years on. http://www.web2summit.com/web2009/public/schedule/detail/ 10194, Accessed 31 Aug 2015.

Richter D., Riemer K., \& vom Brocke J. (2011). Internet social networking: research state of the art and implications for enterprise 2.0. Business \& Information Systems Engineering, 3(2), 1-13.

Riemer, K., Richter, A., \& Böhringer, M. (2010). Enterprise microblogging. Business \& Information Systems Engineering, 2(6), 391-394.

Schlagwein, D., \& Prasarnphanich, P. (2014). Social media around the GLOBE. Journal of Organizational Computing and Electronic Commerce, 24(2-3), 122-137.

Schneider, S., \& Sunyaev, A. (2015). CloudLive: a life cycle framework for cloud services. Electronic Markets, 25(4). In press.

Setia, P., Richardson, V., \& Smith, R. J. (2015). Business value of partner's IT intensity: value co-creation and appropriation between customers and suppliers. Electronic Markets, 25(4). doi:10.1007/ s12525-015-0189-7.

Shirky, C. (2003). Social software: a new generation of tools. Esther Dyson's Monthly Report, 21(5), 1-31.

Steinhueser, M., Richter, A., \& Smolnik, S. (2015). How to bridge the boundary - determinants of inter-organizational social software usage. Electronic Markets, 25(4). doi:10.1007/s12525015-0192-z.

Thompson, C. (2007). The see-through CEO. Wired, 19(4), 134-139.

Williams, S. P., Hausmann, V., Hardy, C. A., \& Schubert, P. (2013). Enterprise 2.0 research: Meeting the challenges of practice. BLED. 